\title{
ANTARCTIC OZONE HOLE AS A NATURAL GEOPHYSICAL OBJECT
}

\author{
Alexander V. Dergunov ${ }^{1}$, Valentin B. Kashkin ${ }^{2}$, Tatyana V. Rubleva ${ }^{2, *}$, \\ Alexey A. Romanov ${ }^{3}$ and Roman V. Odintsov ${ }^{2}$ \\ ${ }^{1}$ Institute of Computational Modeling SB RAS, Krasnoyarsk, 660036, Akademgorodok, Russian \\ Federation \\ ${ }^{2}$ The Institute of Engineering Physics and Radioelectronics of Siberian Federal University, \\ Krasnoyarsk, 660074, Akademika Kirenskogo Str. 28/12B, Russian Federation \\ ${ }^{3}$ The Institute of Space and Information Technology of Siberian Federal University, Krasnoyarsk, \\ 660074, Akademika Kirenskogo Str. 26/1, Russian Federation
}

\begin{abstract}
Satellite data on total ozone content for 1985-2015 have been used. Methods of evaluating ozone deficit in the polar region and its excess in middle latitudes of the Southern Hemisphere have been developed. In early spring the ozone molecules outflow and the ozone anomaly forms. Ozone inflows the middle latitudes, its total content increases and a ring with elevated TO forms. In October-November the dynamic process reverses, from the ring the ozone molecules transfer to the polar latitudes. The amount of ozone leaving the ring into the polar regions and filling the ozone anomaly is virtually the same. The results produces indicate that the Antarctic ozone hole is a natural geophysical formation.
\end{abstract}

\section{Introduction}

The ozone layer protects our planet from the hard ultraviolet rays of the Sun. Total ozone (TO) - is the thickness of gaseous ozone layer in the vertical column of atmosphere at temperature $0^{\circ} \mathrm{C}$ and atmospheric pressure $1013 \mathrm{hPa}$. TO is measured in Dobson units (DU), $1 \mathrm{DU}=10^{-5} \mathrm{~m}$. The ozone anomalies are local regions with substantially reduced total ozone in the Earth's ozone layer below 220 DU.

In 1985 the Southern Hemisphere around the Antarctic in spring was found to form an ozone anomaly (Antarctic ozone hole, $\mathrm{AOH}$ ) [1]. In recent years its area varies from 18 to 30 million $\mathrm{km}^{2}$ [2]. AOH is surrounded by an ring with high TO, its values reach up to 450 DU. In 1986 researchers R.S. Stolarski and M.R. Schoeberl showed that during AOH formation the ozone mass is transferred from the near-polar regions of the Southern hemisphere to the middle latitudes, and from the end of September the ozone mass moves towards the pole. In November-December AOH disappears [3].

\footnotetext{
*Corresponding author: tvrubleva@mail.ru
} 
However, currently is generally accepted the theory holding that the cause of ozone layer depletion in the polar regions of the Southern hemisphere is destruction of ozone by some substances of anthropogenic origin for example chlorfluorcarbons (freons) [4, 5]. Vienna Convention (1985) and the Montreal Protocol (1987) on Substances That Deplete the Ozone Layer were signed. When signing the Montreal Protocol it was claimed that the Antarctic ozone hole would disappear in 2010, however, this anomaly occurs annually.

The work studies redistribution of ozone from the middle latitudes to the polar regions of the Southern Hemisphere in spring 1985-2015 by TOMS (Nimbus-7, EarthProbe, USA) and OMI (Aura, USA) satellite data [6]. Total ozone was measured in the range of 300-380 nm with an accuracy of $2-4 \%$.

\section{The ozone spring dynamics}

Mean zonal TO was analyzed from NASA site [6]. Mean zonal TO are daily values of total ozone in the different 5 degrees rings of latitudes averaged by longitude from 0 to 360 degrees.

To illustrate the dynamic processes running in spring 2013 let us consider plots of mean zonal TO vs. individual days in October and November given in Figure 1. Figure 1, $a$ shows variation of ozone content during the existence of the Antarctic ozone hole. It is evident that over the polar region in October the mean zonal TO values are below the climate normal $220 \mathrm{DU}$. E.g. on the $1^{\text {st }}$ of October in the ring of latitudes $85-90^{\circ} \mathrm{S}$ the zonal TO is 142 DU. In the middle latitudes the total ozone layer substantially exceeds the climate normal and on the $11^{\text {th }}$ of October in the latitude ring $50-55^{\circ} \mathrm{S}$ TO is 353 DU. So in the range of latitudes $45-55^{\circ} \mathrm{S}$ a ring with high the total ozone forms.

Dynamic processes during the existence of $\mathrm{AOH}$ have been considered in [7]. Account is taken of the enormous circumpolar vortex in the Southern Hemisphere reaching over the polar and middle latitudes. In August-November in the stratosphere this vortex is the highpressure area with center in the polar region. Ozone flows (together with the main mass of air) move from the pole in all directions, curving by the Coriolis force from west to east. This forms rotating vortex at the middle latitudes. The vortex absorbs ozone from the polar latitudes and includes the ozone ring with high TO. Ozone concentration within the vortex drastically drops and an area with reduced ozone content including $\mathrm{AOH}$ forms over the pole. By the end of spring the Antarctic stratosphere is heated, the vortex grows into a cyclone, the ozone mass reverses from the ring to the polar region, the anomaly disappears.

Lack of satellite data on ozone content in the polar regions in August-September denies the opportunity to study the ozone mass transfer from the polar area into the middle latitudes. However, these data available for October-November make possible to compare the amount of ozone that has left the polar latitudes and ozone excess in the middle latitudes.

Figure $1, b$ shows variation of zonal TO during active atmospheric processes with $\mathrm{AOH}$ filling in November. We should note that in the polar and middle latitudes these values exceed the climate normal. In the polar regions the ozone content increased, in the middle latitudes it decreased. By the end of November the high TO ring in the middle latitudes disappears (29 November, Figure 1,b), and the ozone hole ceases to exist. 

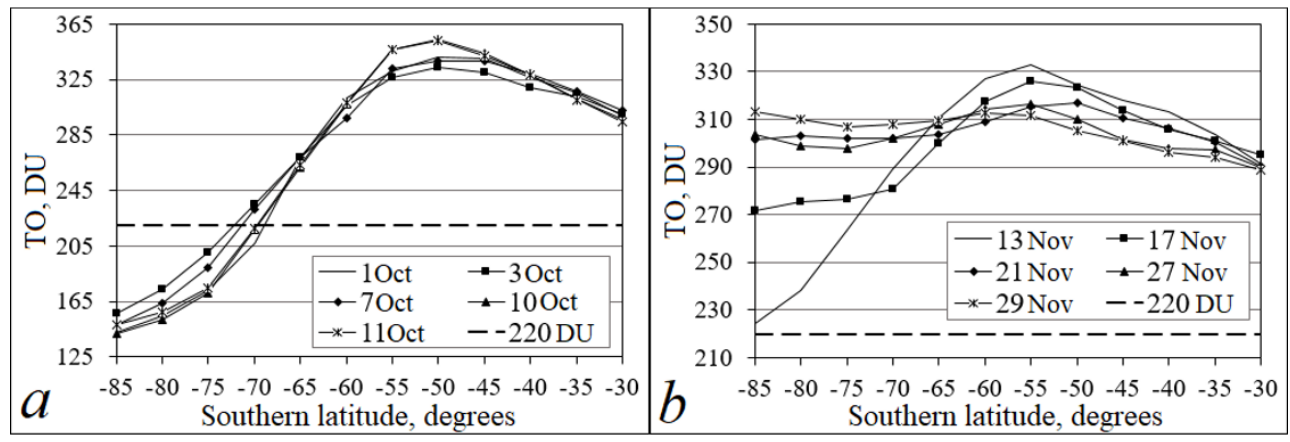

Fig. 1. Variation of ozone content in the Southern Hemisphere in spring 2013.

\section{Select averaging interval}

To improve validity of the analysis we studies a plot averaged zonal TO of vs. latitude over several days. It was necessary to define time averaging interval $\Delta T$.

From the moment of its formation to «filling» the Antarctic ozone hole is known [7] to exist for 3-4 months, i.e. actually from the 20-s of August to mid-December. Zonal data on the total ozone from August till the end of September are not available [6]. During this period the lower stratosphere above the Antarctic is not illuminated by the sun and measurements were not performed. For the beginning of the time interval $\Delta T$ we chose the data for which the NASA base showed the TO data. In 2013 it's the $1^{\text {st }}$ of October.

To define the end of interval $\Delta T$ when the ozone hole «filling» processes start we analyzed deficit of ozone mass $\Delta M$. According to [2] this characteristic is defined as the difference between the ozone mass in $\mathrm{AOH}$ with the boundary of $220 \mathrm{DU}$, and the mass of ozone within actual boundaries of the anomaly. Comparison of zonal TO and $\Delta M$ deficit of $\mathrm{AOH}$ showed that the data of beginning of drastic restoration of the ozone layer in the polar regions in 2013 is the $14^{\text {th }}$ of October. This date was chosen as the end of time interval $\Delta T$. So, in $2013 \Delta T=14$ days.

Figure 2 shows variation of zonal TO in the Southern Hemisphere in two latitudinal zones $75-90^{\circ} \mathrm{S}$ and $45-60^{\circ} \mathrm{S}$ from the $1^{\text {st }}$ to the $22^{\text {nd }}$ of October 2013 . The dash lines delineate boundaries of the earlier chosen $\Delta T$ interval. Figure 2, $a$ indicates income of ozone into the $\mathrm{AOH}$ area. Growth of zonal after the $14^{\text {th }}$ October confirms correct choice of the upper boundary of the interval $\Delta T$.

From the $10^{\text {th }}$ of October the zones of $45-60^{\circ} \mathrm{S}$ lying beyond the polar regions are observed to decrease zonal TO. One can talk about ozone transfer from the middle latitudes into the Antarctic ozone hole. TO is observed to drastically decrease after the $14^{\text {th }}$ of October. So, the data presented in Figure 2 do not contradict the method of choosing the upper boundary of the interval $\Delta T$ in ozone deficit value $\Delta M$ in $\mathrm{AOH}$. 


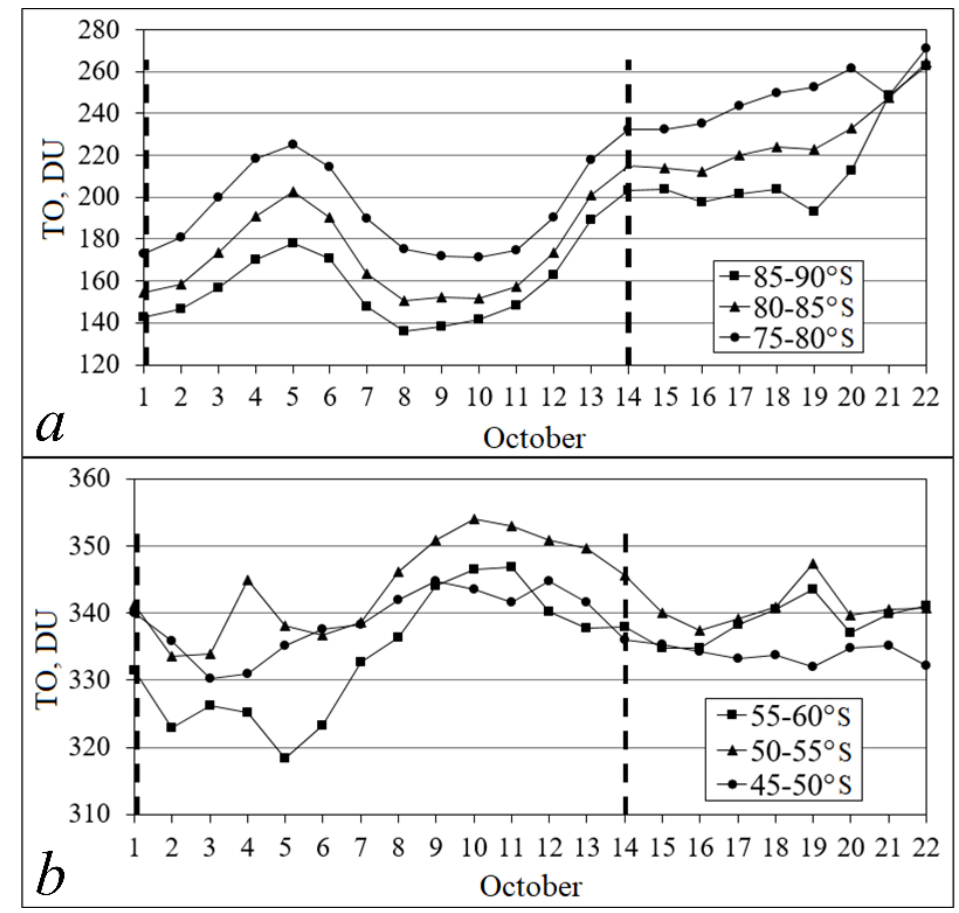

Fig. 2. Variation of zonal TO over interval $\Delta T$ (boundaries of the interval $\Delta T$ are indicated by the dash lines) for the polar latitudes $(a)$ and for the middle latitudes $(b)$.

\section{Results analysis}

Let us compare the amount of ozone that has «left» the polar regions and ozone excess in the middle latitudes. For this we need to define ozone excess in the middle latitudes and its deficit in the polar regions. For this we choose some threshold value of TO, denote it as $X_{0}$. Exceeding the $X_{0}$ values correspond to the excess, and below the $X_{0}$ threshold we observe deficit. The threshold value of $X_{0}$ it was experimentally found to be the annual mean total ozone content in the range of latitudes from $32.5^{\circ}$ to $52.5^{\circ} \mathrm{S}$ for every year. According to the NASA database all zonal mean are present in this latitude range without omissions. For 2013 the threshold value of $X_{0}=295.5$ DU.

Figure 3 shows deviation of ozone content in the polar and middle latitudes of the Southern Hemisphere from $X_{0}$ threshold in the chosen interval $\Delta T$. In Figures $3, a$ and $3, b$ the threshold value of $X_{0}$ is indicated by the dash line. The plot presented in Figure 3, $a$ matches the plot in Figure 1, $a$ with the only difference that the plot in Figure 1, $a$ represents dependence of daily zonal means vs. geographic latitude $\varphi$, while the plot in Figure 3, $a-$ daily zonal means are averaged over $1-14$ October. Denote the zonal values averaged in this way by $x_{\varphi}$. In Figure 3, $a$ it is evident, that the amount of ozone $x_{\varphi}$ in the polar region is less than the threshold $X_{0}$ at the latitudes above $63^{\circ} \mathrm{S}$. This area might be specified by ozone deficit, and in Figure 3, $a$ it is denoted by «-». In Figure 3, $a$ at the latitudes below $63^{\circ} \mathrm{S}$ the amount of ozone $x_{\varphi}$ exceeds the threshold TO. This area is specified by ozone excess, it is denoted by «+». 


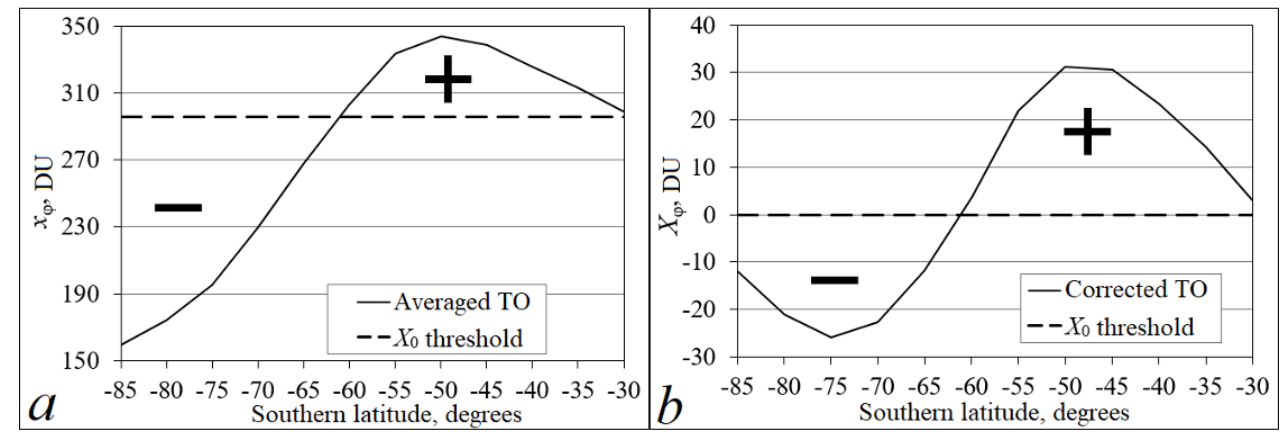

Fig. 3. Deviation of ozone content from the $X_{0}$ threshold in the polar and middle latitudes of the Southern Hemisphere.

In Figure 3, $a$ it can be seen that the areas «-» and «+» differ in size. Here the spherical shape of the Earth is not taken into account. The zonal TO should be multiplied by the cosine of latitude $\varphi$ for sphericity correction: $X_{\varphi}=x_{\varphi} \cos \varphi, X_{\varphi}$ is corrected value of TO.

Denote deviation of the corrected value of $X_{\varphi}$ from the threshold as $\Delta X_{\varphi}=X_{\varphi}-X_{0}$.

Dependence of $\Delta X_{\varphi}$ values on the latitude is given in Figure 3,b.

Sum up deviations from the threshold $X_{0} \Delta X_{\varphi}$ separately with for the area with «-» sign and for the area with знаком «+» sign. In the first case the sum of $\Delta X_{\varphi}$ values specifies ozone deficit $D$ in the polar area. In the second - the sum of $\Delta X_{\varphi}$ values for the area with «+» sign it is excess of ozone $E$ in the middle latitudes.

Results of zonal TO studies for 1985-2015 are presented in the Table. The first column is the years, the second - threshold $X_{0}$, deficit $D$ is the third column, excess $E-$ in the fourth. About the difference $Q$ of deficit and excess one can judge from the last column which shows this difference in per cent. Value $Q$ is calculated by formula:

$$
Q=\frac{D-E}{(D+E) / 2} \text {. }
$$

\begin{tabular}{|c|c|c|c|c|}
\multicolumn{7}{|c|}{ Table } \\
\hline Year & $X_{0}$, Du. & $D$, Du. & $E$, Du. & $Q, \%$ \\
\hline 1987 & 305.7 & 151.8 & 153.6 & -1.2 \\
\hline 1989 & 312.9 & 149.1 & 152.4 & -2.2 \\
\hline 1990 & 304.4 & 138.1 & 135.4 & 2.0 \\
\hline 1991 & 311.2 & 130.5 & 203.5 & -43.7 \\
\hline 1999 & 299.2 & 161.5 & 151.6 & 6.3 \\
\hline 2005 & 302.3 & 128.5 & 130.4 & -1.4 \\
\hline 2010 & 295.7 & 121.7 & 115.9 & 4.9 \\
\hline 2013 & 295.5 & 89.7 & 98.1 & -8.9 \\
\hline 2014 & 302.8 & 123.6 & 129.8 & -4.9 \\
\hline 2015 & 296.5 & 198.3 & 94.5 & 70.9 \\
\hline
\end{tabular}

The estimates given in the Table show that the deficit of ozone $D$ in the polar areas and its excess $E$ in the middle latitudes practically coincide, and value $Q$ is of the same order as the measurement error.

The exception are years 1991 and 2015, when $Q$ was $-43.7 \%$ and $70.9 \%$, respectively. This seems to be associated with the action of Pinatubo (1991) and Calbuco (2015) volcanoes. During volcanic activity eruption products (e.g. sulfur dioxide) are emitted and can destroy the ozone layer [8]. Eruption of Pinatubo volcano $\left(15.07^{\circ} \mathrm{N} ; 120.21^{\circ} \mathrm{E}\right)$ 
occurred in the Philippines in 12-17 June1991 was with Volcanic Explosivity Index VEI = 6. Eruption of Calbuco volcano $\left(41.33^{\circ} \mathrm{S} ; 72.61^{\circ} \mathrm{W}\right)$ with VEI $=4$ occurred in Chile $22-30$ April 2015. These events might partially deplete the ozone in the Southern Hemisphere and result in the mismatch of the ozone deficit in the polar regions and its excess in the middle latitudes.

Regretfully we did not succeed to analyze the matching of ozone deficit and excess for every year. The main case was partial or complete lack of zonal data on TO. For 1993 1995 these data are completely absent. For 1985, 1986, 1992, 1996, 1997, 1998, 2002, 2003, 2007, 2008, 2011 omissions in the zonal data up to 33 days. This denied correct choice of $\Delta T$ time interval averaging and defining the threshold $X_{0}$.

\section{Conclusion}

Methods of evaluating the ozone deficit in the polar areas and its excess in the middle latitudes of the Southern Hemisphere during existence of the Antarctic ozone hole have been developed. The essence of the method is to compare the amount of ozone in the polar and middle areas with a certain threshold value. Mean annual total ozone content in the latitude range from $32.5^{\circ}$ to $52.5^{\circ} \mathrm{S}$ for every year was experimentally found to be the threshold value. Estimates have been made to show that during the existence of the Antarctic ozone hole the ozone deficit in the polar areas and its excess in the middle latitudes of the Southern hemisphere practically coincide. Estimates of the difference between the deficit and the excess in most cases are of the same order as the measurement error.

Our studies confirm the results of R.S. Stolarski and M.R. Schoeberl and indicate that the Antarctic ozone hole is a natural geophysical formation. We believe that the theory of the origin of the hole from chemical ozone depletion is not correct.

\section{References}

1. J.C. Farman, B.G. Gardiner, and J.D. Shanklin, Nature, 315, 207 (1985)

2. NASA Ozone Watch, Images, data, and information for atmospheric ozone, http://www.ozonewatch.gsfc.nasa.gov

3. R.S. Stolarski, M.R. Schoeberl, Geophys. Res. Lett., 13, 1210 (1986)

4. S. Solomon et al., Nature, 321, 755 (1986)

5. S. Solomon et al., Science, 353, 269 (2016)

6. NASA, Ozone, ftp://jwocky.gsfc.nasa.gov/pub/

7. V.B. Kashkin, T.V. Rubleva, and R.G. Khlebopros, Stratospheric ozone: space orbit view (Krasnoyarsk, SFU, 2015)

8. V.V. Zuyev, N.Ye. Zuyeva, Optics of the atmosphere and ocean, 24, 30 (2011) 